\title{
Remote Patient Management in Home Dialysis: Planning Considerations for the Future
}

\author{
Michael Whitlow • Eric Wallace
}

Department of Medicine, Division of Nephrology at the University of Alabama, Birmingham, AL, USA

\begin{abstract}
Remote patient management (RPM) has the potential to improve patient outcomes in home dialysis patients. Achieving this requires an operational plan that addresses data collection, RPM adoption, and plans for therapeutic interventions on abnormal results. The objective of this chapter is to discuss the key factors to be considered when setting up a RPM program, and in particular how a RPM program can be beneficial in the management of home dialysis patients. RPM is already being utilized in many other disease states to a greater extent than the home dialysis population (heart failure, COPD, diabetes) to improve clinical outcomes. The key components needed to set up a RPM program will be discussed in this chapter. Furthermore, literature regarding the potential benefits to the home dialysis population will be reviewed along with barriers to the adoption of technology.

(c) 2019 S. Karger AG, Basel
\end{abstract}

\section{Introduction}

Remote patient management (RPM) is an evolving technology that allows for real-time examination of a patient's clinical status from their home. Making use of biotech sensors and data from remote patients, RPM technology transmits clinical information collected by patients to healthcare providers in real time, allowing unique insight into a patient's clinical status between clinic visits. Blue- 
tooth-enabled blood pressure cuffs, scales, oxygen saturation monitors, and data including dialysis treatment parameters can be collected and sent for review by medical staff. The access to this information has the potential to guide therapeutic interventions to prevent deterioration of clinical status. The use of RPM has the potential to improve patient outcomes, improve patient uptake to home therapies, and improve technique survival. However, many questions about appropriate thresholds, interventions, and operations of a RPM program exist. This chapter will focus on the potential benefits and challenges that RPM holds for the PD population.

\section{Starting a RPM Program}

\section{What Should Be Monitored?}

Before implementation of a RPM program, it is important to plan ahead. All team members including nursing staff, physicians, and ideally patients should be included. Details such as what data are going to be monitored, how often the data will be reviewed, and what target thresholds to trigger intervention should be discussed before implementation. RPM programs, although largely revolving around Bluetooth-enabled devices such as blood pressure cuffs and scales as examples, could also entail monitoring of patient captured dietary parameters and changes in answers to survey questions. RPM could start with monitoring of only one parameter or multiple parameters. However, with every parameter measured comes more data, which if not planned appropriately could overwhelm providers and limit adoption.

It is still unknown what parameters are the most meaningful to be monitored in the home dialysis population. Parameters such as blood pressure and weight should be useful in determining the volume status. Other markers of volume such as bioimpedance and body volume water have largely not been successful in improving the clinical outcomes [1]. However, multiple repeated measurements in real-time might improve the utility bioimpedance. Oxygen saturation may be a useful metric to follow as nocturnal hypoxemia has been shown to correlate with cardiovascular complications in the ESRD population [2]. New wearables, such as smart watches, can be beneficial as they can monitor the acute changes in heart rate, development of new arrhythmias, and hyperkalemia [3]. Remotely collected glucose data could also prove useful, specifically in the PD population. Dialysis therapy parameters and alarms may also be useful to predict catheter malfunction and ensure compliance with therapy. Remote patient management can also ensure the collection of treatment-related data which in the United States is required for insurance and regulatory purposes. Patient report- 
Table 1. What can we monitor?

\begin{tabular}{|c|c|}
\hline Disease & RPM data \\
\hline COPD & $\begin{array}{l}\text { Daily spirometry (FEV }, \text { FVC, PEF) } \\
\text { Inspiratory capacity } \\
\mathrm{SpO}_{2}\end{array}$ \\
\hline Heart failure & $\begin{array}{l}\text { Weight } \\
\mathrm{SpO}_{2} \\
\text { Vitals (heart rate, } \mathrm{BP} \text {, etc.) } \\
\text { Invasive monitors (utilizing invasive devices such as PPM to } \\
\text { monitor for arrhythmias, intrathoracic impendence, and patient } \\
\text { activity level) } \\
\text { CardioMEMS }\end{array}$ \\
\hline Diabetes mellitus & $\begin{array}{l}\text { Automatic recording of glucose levels } \\
\text { Vitals (weight, BP, heart rate, etc.) } \\
\text { Waist circumference }\end{array}$ \\
\hline Peritoneal dialysis & $\begin{array}{l}\text { Remote data sent from PD cycler (treatment time, UF total, } \\
\text { compliance) }\end{array}$ \\
\hline Home hemodialysis & $\begin{array}{l}\text { Remote data sent from home HD machine (UF rate, weight, } \\
\text { blood pressure, medications) }\end{array}$ \\
\hline
\end{tabular}

ed data such as questionnaires on quality of life, symptomatology, or pain may also prove useful to trigger early intervention in depression or mental illness (Table 1).

How does a program choose a parameter that if measured would prove the most time efficient and cost effective? More studies are needed to determine which parameter, if monitored, is most useful in ensuring outcomes. Many RPM based on platforms can run algorithms to risk stratify patients into low, medium, and high-risk populations predefined algorithms allowing for easy identification of patients in need of assistance; however, data to validate these algorithms are lacking in the home dialysis population.

\section{How Often and How Long Should Patients Be Monitored?}

It is important to set goals ahead of time as to how often data will be evaluated. Most RPM programs for home dialysis are not intended to be reviewed in real time. The frequency of data collection and the review of data should be discussed with the patient in advance, and if indicated a signed consent should be obtained. Many home dialysis patients are very stable and might not need intense monitoring. In general, data can be reviewed once a day by nursing staff. There has been some investigation regarding the frequency of monitoring RPM data in the heart failure population as one study found a reduction in hospital read- 
missions with daily physiological monitoring. However, other studies have shown an equivalent improvement in outcomes with less frequent monitoring coupled with monthly nursing calls. Overall, more studies are needed to further investigate the ideal frequency of monitoring [4].

How long will patients be on the program? It should be noted that most RPM programs do not monitor patients forever. Limiting data collection to 3 months depending on the situation is standard. However, in the case of home dialysis, the regulatory benefit of getting all the treatment data as well as having accurate information on which to make therapeutic adjustments in therapy might lend itself to lifelong remote monitoring but without daily review on these patients, reviewing the parameters once monthly instead. This approach would limit the nursing time while still allowing for the dialysis unit and patient to benefit from the program.

\section{Who Should Be Monitored?}

Monitoring every home dialysis patient may inundate nurses and ultimately not improve outcomes. Studies of RPM have shown that most efficient monitoring is done by preselecting a higher risk patient population. Who is this population in home dialysis? Previous work in heart failure, DM, and COPD has focused on patients with multiple comorbidities or patients with more severe disease (e.g., NYHA III symptoms or severely reduced FEV1). However, comorbidities alone, being so common in the dialysis population, may not be adequate as a distin-

guishing feature. Studies are needed to determine the patient population that would require more intense RPM.

One example of a high-risk group are patients who have been hospitalized. Patients post hospitalization should be monitored to prevent readmissions. Previous studies have evaluated the impact RPM can have in other disease states on improving post-hospitalization care. The use of RPM in COPD has been shown to reduce admission rates as well as a total decrease in hospital days and outpatient visits [5]. In the CHAMPION study, NYHA class III HF patients were randomized to either RPM monitoring system (wireless implantable hemodynamic monitoring system) or usual care. The RPM group experienced a reduction in hospital admissions for HF by $30 \%$ for up to 6 months. The duration of hospital stay was also reduced. [6]. Hence, PD patients with heart failure and particularly NYHA III symptoms might be a subset of the PD population that might benefit from RPM. Overall, more work is needed to determine exactly which patients need to be monitored but it is the opinion of the authors that a RPM program should start monitoring new patients, any patients hospitalized for volume-related issues, and patients with any question of compliance at a bare minimum. 


\section{How to Monitor?}

Once the targeted metric is identified and details of the program are planned, planning for the equipment must occur. What platform will be purchased? Does the vendor meet the IT security requirements of your institution? Is there a possibility of an interface with your electronic medical record to consolidate log in's for staff.

What is the workflow for the patient? Data collection must be as easy as possible for the patient. Automated data entry is preferred to data entry requiring the patient to manually enter data. Furthermore, you must know the technology literacy as well as the average access to Internet in your patient population. Some remote monitoring platforms require each and every piece of equipment to be paired with the hub device. This has advantages as it is more flexible on the types of devices that can be used, allowing for different brands of sensors in the future. However, there are disadvantages as well in that more nursing and/or patient time/knowledge is required to get the program set up and having to pair each of the devices. Other vendors have pre-paired devices which saves on time and gives more of a plug-and-play feel but limits the devices being used.

Another consideration is internet connectivity. It should not be assumed that most patients have internet connectivity. The patients least likely to have adequate bandwidth are older, socioeconomically disadvantaged, and rural [7]. Limiting transmission of data to $\mathrm{WiFi}$ will greatly reduce the amount of patients able to be monitored. Some RPM systems will transmit over cellular but cellular coverage, although robust, still has gaps and can be limited further by roofing materials such as metal. Finally, there are systems that have the option of transmitting data over either WiFi, cellular, or standard landline transmission. This is possible as RPM data, as it does not necessarily involve video, usually does not require large bandwidth to transmit data.

\section{Why Monitor? Benefits of RPM}

Improving Patient Education

Education is a critical component of PD. Patients must learn how to set up their PD treatment and also know some basics of how to troubleshoot different problems. Patients also need to be able to recognize when they need to contact their PD nurse about particular issues. RPM can improve education, as nurses will contact patients when they recognize abnormalities in the data that are being transmitted via RPM. Subsequently, the patient learns which parameters to monitor more closely and can perform the necessary interventions or contact their nurses sooner. 
Reducing Travel Burden

Travel is a major barrier for many patients when it comes to receiving good healthcare. Patients will often use travel distance as an excuse for missing appointments, which ultimately leads to substandard care. Travel particularly hinders some peritoneal dialysis (PD) patients as they drive on average a farther distance to their home dialysis unit compared to in-center hemodialysis patients driving to their units [8].

Frequent home visits are one way to reduce the burden of travel. Frequent patient-provider interaction has been shown to cause a reduction in both causespecific hospitalizations and recurrent hospitalizations [9]. Frequent patientprovider interaction can also lead to a reduction in attrition from home dialysis therapy and thus ensure that patients are compliant and feel comfortable using home therapies [10]. However, increasing patient-provider interactions through home visits can be expensive. RPM can help to reduce the frequency the patients need to be evaluated as clinical information can be monitored remotely in between visits. Overall, a combination of telehealth, through videoconferencing, as well as use of RPM can help to reduce the time and cost burden associated with both travel to outpatient clinics and the need for home visits.

\section{Managing Supplies}

Supplies are another critical aspect of PD. It is important that patients have their $\mathrm{PD}$ equipment readily available to make sure they do not miss any dialysis sessions and can make adjustments in their PD prescription if necessary. Data from Baxter have shown that late orders will occur in approximately $10 \%$ of home PD patients per month. The delay in the delivery of supplies not only endangers the patient's health but can also lead to increased cost due to the need for emergency delivery of supplies [8]. RPM could allow for the ability to monitor a patient's supplies and make sure that the patient's PD supply inventory is consistently stocked. Ultimately better management of PD supplies can lead to improved care and reduction in cost burden by reducing the need for emergency deliveries.

\section{Managing Data}

Data collection is a major aspect of home dialysis therapy. Patients will spend a significant amount of time collecting and recording a variety of clinical information including weights, total UF value, vitals etc. Subsequently, physicians and nurses can then spend a significant amount of time reviewing and analyzing this information. Through Bluetooth technology, RPM can provide an efficient way to collect patient data without the need for the patient to manually record the data. Real-time monitoring of different clinical variables can improve the efficiency of care doctors and nurses can provide to address home dialysis patients. 


\section{Compliance}

Compliance is another critical aspect of home dialysis therapy. One of the unique aspects of home dialysis is the responsibility it places on patients to make sure they perform their dialysis therapy as prescribed. At in-center hemodialysis clinics, physicians can monitor whether a patient has been coming for their dialysis treatments to determine if they are compliant. However, with home dialysis, physicians are usually only able to obtain information regarding compliance at the monthly clinic visits. The onus placed on the patient to collect their data on flow sheets only further complicates a physician's job of determining whether patients are receiving adequate treatments. Additionally, compliance has been shown to be an indicator for risk of hospitalization as well as risk for developing peritonitis. RPM can provide an opportunity to monitor whether patients are performing their PD as prescribed and allow physicians to intervene earlier before a patient has any problems.

\section{Impact on Cost}

RPM can have a significant impact on reducing a patient's financial burden. Additionally, RPM can also reduce the financial burden on the healthcare system in general. The direct impact of RPM to cost has been evaluated. In one study, RPM was used to monitor blood pressure and weight in PD patients. The use of RPM to oversee patient's weight was found to result in fewer hospitalizations as well as fewer days hospitalized [11].

Another simulated study looked at the impact RPM could have on hospitalization rates as well as on cost. The study found an overall reduction in one to 2 hospitalizations, 1-4 home visits, and 2-5 emergency department visits. Overall, the reduction in healthcare utilization in this simulated study showed a potential for a total savings of USD 23,000 [12]. The use of RPM in heart failure has been shown to cause a significant reduction in hospital length of stay. Subsequently, this reduction in length of stay reduces the cost associated with hospitalization. In this study, the difference in cost per hospitalization in the group under RPM monitoring versus standard care patients was USD1,793 when taking into account the cost of the monitoring device [13].

\section{Barriers to Implementation of RPM}

Despite the many benefits of RPM, there are several barriers. There are many different clinical parameters that can be monitored to help improve clinical efficiency. However, one must still determine which clinical variables are the most critical and which will ultimately lead to an improvement in clinical outcomes. Additionally, once data become available, physicians and nurses will then have 
to sift through large amounts of data placing a possibly unreasonable burden on the healthcare provider.

RPM also carries its own cost burden. The cost associated with RPM includes cost of setting up a program, labor cost associated with nursing time to evaluate and organize the data, and inconsistent forms of reimbursement for RPM. Having an appropriate plan to intervene and act on the clinical information received is also important. It is possible that continuous monitoring of patients without an effective intervention plan for the data received could lead to increased hospitalizations and thus increased cost [8].

As mentioned, reimbursement is another barrier to RPM in the home dialysis population. In the United States, Medicare does provide reimbursement for RPM in many disease states such as heart failure, COPD, and diabetes mellitus. Physicians can be compensated for the time it takes to set up and educate patients about RPM as well as the time clinical staff spend evaluating and intervening on remote patient data. However, ESRD patients are all excluded from these billing mechanisms, thus limiting the incentive for physicians to start RPM programs for home dialysis patients in the United States.

\section{Possible Solutions to RPM Barriers}

To reduce the labor and cost burden associated with evaluating large amounts of data that comes with RPM, it will be important to develop algorithms to help with data management. A physician can use these algorithms to flag certain clinical parameters or patient complaints that would require direct interpretation or action. Therefore, the physician and nurses would only have to respond to the most critical information, which could make the process much more efficient.

Artificial intelligence (AI) is a burgeoning area of technology that is being used in many different fields, including medicine. One benefit of AI is the ability to organize and evaluate large amounts of data. AI has been utilized to help physicians find vital patient information quickly through a chat interface instead of having to spend time searching through a patient's chart. Per the American College of Physicians, providers can spend up to 50\% of their time looking for information in a patient's electronic medical record. AI could help to reduce this burden on the physician and lead to more effective clinical care [14]. Just as AI can help physicians with the management of EMR data, it could also be helpful for RPM. RPM can present providers with large amounts of data. Subsequently, the issue becomes the time healthcare providers have to take to evaluate all this data. AI could help providers systematically organize all this data and determine what data needs to be acted upon. 


\section{Conclusion}

RPM is an important tool that physicians have to improve the efficiency at which care is being delivered in many different disease states including PD. RPM can help reduce the burden of travel as well as improve the process of managing PD supplies. Additionally, the ability to receive vital patient information in real time allows the physician to directly impact a patient's care sooner and hopefully lead to fewer complications. Overall, the potential opportunities and promising impact RPM could have on PD is exciting and could lead to an expansion of the number of patients receiving home dialysis as well as making sure the care they receive is as optimal as possible.

\section{References}

1 Lyons KJ, Bischoff MK, Fonarow GC, Horwich TB: Noninvasive bioelectrical impedance for predicting clinical outcomes in outpatients with heart failure. Crit Path in Cardiology 2017;16: $32-36$.

2 Zoccali C, Mallamaci F, Tripepi G: Nocturnal hypoxemia predicts incident cardiovascular complications in dialysis patients. J Am Soc Nephrol. 2003;13:729-733.

3 FDA Designates AliveCor's Bloodless Hyperkalemia Test a "Breakthrough Device." https://www. alivecor.com/press/press_release/fda-designatesalivecors-bloodless-hyperkalemia-test-a-breakthrough-device/ (September 10, 2018).

4 Chaudhry SI, Phillips CO, Stewart SS, Riegel B, Mattera JA, Jerant AF, Krumholz HM: Telemonitoring for patients with chronic heart failure: a systematic review. J Card Fail 2007;13:56-62.

5 Trappenburg JC, Niesink A, de Weert-Van Oene GH, van der Zeijden H: Effects of telemonitoring in patients with chronic obstructive pulmonary disease. Telemed J E Health 2008;14:138-146.

6 Anker SD, Koehler F, Abraham WT: Telemedicine and remote management of patients with heart failure. Lancet 2011;378:731-739.

7 Reiland R: Lack of Broadband Limits Telemedicine in Rural Areas. http;//www.nextavenue.org (March 15, 2018).
8 Wallace EL, Rosner MH, Alscher MD, Schmitt CP, Jain A, Tentori F, Firanek C, Rheuban KS, Florez-Arango J, Jha V, Foo M, de Blok K, Marshall MR, Sanabria M, Kudelka T, Sloland JA: Remote patient management for home dialysis patients. Kidney Int Rep 2017;2:1009-1017.

9 Prakash S, Coffin R, Schold J, Lewis SA, et al: Travel distance and home dialysis rates in the United States. Perit Dial Int 2014;34:24-32.

10 Slinin Y, Guo H, Li S, et al: Association of provider-patient visit frequency and patient outcomes on hemodialysis. J Am Soc Nephrol 2012;23: 1560-567.

11 Lew SQ, Sikka N, Thompson C, Magnus M: Impact of remote biometric monitoring on cost and hospitalization outcomes in peritoneal dialysis. J Telemed Telecare 2018;1.

12 Makhija D, Alscher M, Becker S, D’Alonzo S, Mehrotra R, Wong L, McLeod K, Danek J, Gellens M, Kudelka T, Sloand J, Laplante S: Remote monitoring of automated peritoneal dialysis patients: assessing clinical and economic value. Telemed J E Health 2018;24:315-323.

13 Crossley GH, Boyle A, Vitense H, Chang Y, Mead $\mathrm{RH}, \mathrm{CONNECT} \mathrm{Investigators:} \mathrm{The} \mathrm{CONNECT}$ (Clinical evaluation of remote notification to reduce time to clinical decision) trial the value of wireless remote monitoring with automatic clinician alerts. J Am Coll Cardiol 2011;57:1181-1189.

14 Pacis D, Subido E, Bugtai N: Trends in Telemedicine Utilizing Artificial Intelligence. AIP Conference Proceedings, 2018.

Eric Wallace, MD

Department of Medicine, Division of Nephrology at the University of Alabama

JNWB 416, 500 22nd Street South

Birmingham, AL 35294 (USA)

E-Mail elwallace@uabmc.edu 